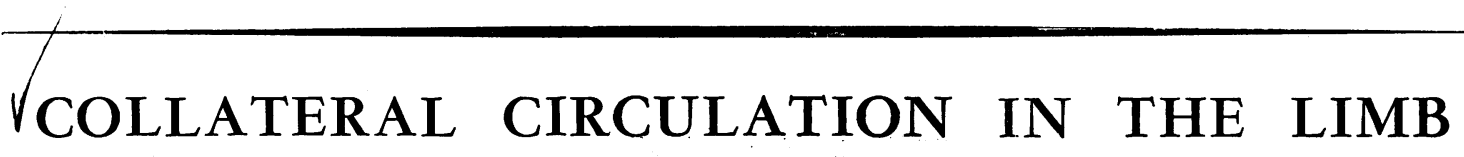

By C. J. Longland, M.V.O., M.S., F.R.C.S.

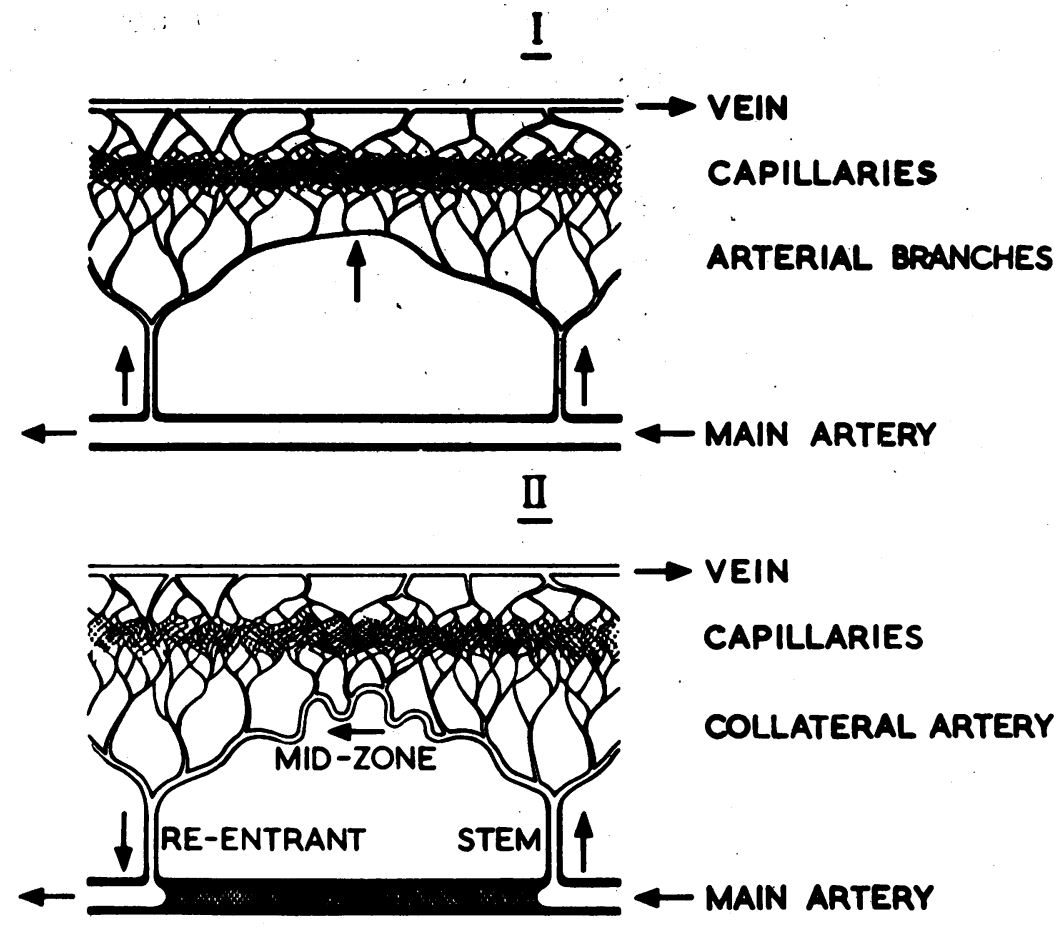

The most important consequence of all varieties of arterial disease is obliteration of the arterial lumen. If tissues are to survive after arterial block, the blood flow must be restored through other channels. The remarkable metamorphosis into collateral arteries which these alternative pathways undergo has its roots in the potentialities for growth of adult blood vessels; as a compensatory mechanism this ranks with, for example, the striking compensatory hyperplasia met with in the liver.

It is axiomatic that an adequate arterial system is vital to the existence and function of limb tissues, but it may perhaps be less well appreciated that the capacity of arterial diseases to interfere with the flow of blood by occluding arteries depends in very large measure on the effectiveness of the collateral response. So regarded, the formation of collateral circulations appears as one of the most important aspects of arterial disease.

\section{Collateral Pathways}

The accompanying diagram (Fig. I) depicts the conversion of collateral pathways in relation to a o main artery into a collateral artery after obstruction of the main vessel. This collateral artery is derived from two major branches and intermediate anastomotic vessels, and these parts have been 0 labelled the 'stem,' ' mid-zone' and ' re-entrant' components.

Potential collateral pathways of the kind illus- $\rightarrow$ trated are plentiful in the limbs; there are frequent branches from the main vessels which break up to N supply various tissues; in the case of muscle, which is rich in arteries, anastomoses are plentiful 0 and sometimes of a size visible to the naked eye; $N$ the skin is irrigated by a network of arteries, and anastomotic arterial rings are formed about the 0 joints. The other soft tissues have fewer vessels, $\frac{D}{\mathbb{D}}$ but these form anastomotic connections in the $\stackrel{?}{+}$ same way. Anastomotic systems are, in short, 
present throughout the limbs but vary in their calibre and number in different regions (Woollard, 1934; Salmon, 1933, 1936, 1938).

Important regions poor in collateral pathways are those about the brachial and femoral arteries above their deep (profunda) branches, and the popliteal artery. Anatomical investigation suggests, and clinical experience (de Bakey and Simeone, 1946) demonstrates, that sudden occlusion of these arteries, as by ligature, is fraught with danger of gangrene in a proportion of limbs, so poor may these collateral pathways be. There is also evidence that collateral pathways become less numerous as age advances; nevertheless, large collateral arteries can be developed from them, even in old people.

\section{The Process of Enlargement}

Obstruction of a main artery instantly causes important changes in the flow of blood through neighbouring vessels, including collateral pathways.

I. Physical changes. These may be studied in a model made from rubber tubing. The physical changes taking place in such an inert elastic collateral vessel, transmitting water from a source at constant pressure, when the main vessel is occluded, are :

(a) The lateral pressure in the collateral falls.

(b) In consequence its calibre decreases.

(c) The pressure gradient between the ends of the collateral rises.

(d) The volume flow through the collateral accordingly increases.

Similar changes in pressure gradient and flow can be demonstrated in vivo (Dornhorst and Sharpey-Schafer, 195I).

2. Biological changes. Since it appears that, at least in some circumstances, the physical consequence of main vessel block is to narrow collateral channels, their characteristic enlargement must be an active, vital phenomenon. Thoma, in I893, from his studies of the development of vessels in the area vasculosa of the chick embryo, put forward the principle that increase in size of the lumen of a vessel is dependant on the rate of blood flow through it. Hughes (1935, 1937) made further studies of the chick's area vasculosa and concluded that enlargement of its embryonic capillaries depended on the velocity of the blood stream in contact with the endothelium. $\mathrm{He}$ suggested that changes in velocity altered the stresses in the endothelial cells and that these stresses modified their growth.

That the velocity of the blood current may also provide the stimulus to enlargement of collateral arteries in the adult is suggested by arteriographic observations in full-grown rabbits. Serial arteriograms after obstruction of the femoral artery enable the development of the related collateral systems to be observed. Such arteriograms show that the mid-zone of a collateral artery is often very small initially in comparison with the stem and re-entrant components, but that as time goes on an artery of fairly uniform calibre is evolved. That is, the narrow mid-zone grows proportionately faster than the wider ends of the collateral; if little or no blood escapes from the collateral through side branches, and such is almost certainly the case, then the velocity of the blood stream will be greatest in the narrowest reaches. Thus, here too, growth is most rapid where the velocity is highest as in the embryo.

Serial arteriography shows the order of events in the development of a collateral system to be as follows: Within half an hour of obstruction of the rabbit's femoral artery fine vessels make their appearance in the thigh; in the next few days numerous fine collateral channels are visible and the mid-zones exhibit signs of lively activity, namely, rapid increase in diameter and also in length, resulting in a wavy outline. As the months pass certain of these channels enlarge more than their fellows and at the end of a year relatively few, large, collateral arteries have been evolved; they have fairly uniform calibres but show much undulation. Other vessels, also originally prominent, later fail to show and have presumably undergone regression. The final pattern is the resultant of this interplay of enlargement and regression among the numerous vessels called into play to meet the emergency of the block.

Collateral systems do not compensate completely for obstruction of a main artery. Experimentally, ligature of the femoral artery in the dog causes a prompt fall of pressure beyond the ligature, and though the pressure soon begins to recover as the collaterals enlarge, it is not fully restored even after many months, when growth is presumably complete (Eckstein, Gregg and Pritchard, 1941). A familiar manifestation of this failure to achieve full compensation is the persistence of exercise pain for long periods after arterial block. Such limited collateral development may be due either to some inherent limit of growth being reached or to failure of the growth stimulus; in the latter case many or all of the vessels remain capable of further enlargement in response to appropriate stimulation. When collateral vessels, at first enlarged, are later found to regress, as mentioned above, the growth stimulus has failed and there is evidently a collateral reserve still undeveloped in existing circumstances.

\section{Vaso-Motor Control}

It may seem odd that an intact autonomic system should exercise a restraining influence on a process as vital as the opening up of collateral 
pathways. Yet such appears to be the case. It has been shown in animals (Theis, 1933) and man (Dornhorst and Sharpey-Schafer, 1951) that after ligature of a main artery the resistance to the flow of blood through the collateral channels is reduced if the vaso-motor nerves are interrupted. In the rabbit's thigh lumbar sympathectomy usually causes an immediate increase in size of developing or mature collateral vessels.

Thus, though modified from their normal forebears in that they have undergone very considerable growth and though they may be under a powerful stimulus to enlarge, collateral arteries retain the response of normal arteries and dilate on denervation. This is followed by a regain of tone, and hence a tendency to return towards their original diameters. Clinical experience of improvement in patients with exercise pain after lumbar sympathectomy indicates that the regained diameters are often greater than those prevailing before sympathectomy; furthermore, collateral resistance can be 'shown to remain lower in some patients for at least three months after sympathectomy.

It is probable that two distinct mechanisms operate to promote collateral enlargement after sympathectic denervation:

I. There is dilatation of collateral vessels due to the removal of nervous constrictor tone from their walls.

2. There is an increase of blood flow in the denervated part consequent on dilatation of arterioles; this increased blood flow is of necessity transmitted by the collateral vessels and stimulates them to enlarge.

Both mechanisms are affected by the subsequent regain of tone and it is difficult to determine the relative importance of each.

\section{Assessment of Collateral Circulation}

When a main artery becomes blocked the blood reaching the periphery is transmitted by the collateral system and by any other main vessels which are intact. The collateral circuit introduces an abnormal resistance to the blood flow which tends to be reduced in proportion to the added resistance. The adequacy of the collateral system can therefore be gauged by familiar clinical signs related to the state of the peripheral blood flow. These include the state of nutrition of the distal tissues, the colour and temperature of the skin and the presence or absence of pain at rest or on exercise. The patient should be examined in a warm room to avoid thermo-regulatory reduction of the peripheral blood flow.

The volume of blood flowing through portions of the limb may be measured by the venous occlusion plethysmograph; if the arteriolar resistance is reduced to a low level by, for example, heat, exercise or the induction of reactive hyperaemia the flows measured are largely governed by the collateral systems and, by comparison with $\stackrel{\square}{\complement}$ normals, afford some index of their efficiency.. . Simultaneous measurement of the distal blood $\vec{\Rightarrow}$ pressure (the systemic B.P. being known) permits $\stackrel{\text { क }}{+}$ of exact calculation of the collateral resistance. Though arterial pulsation is not related to blood $\overline{\bar{D}}$ flow, restoration of pulsation to arteries beyond $a \stackrel{\bar{D}}{\bar{D}}$ block, as shown by palpable pulses or good $\mathscr{\Phi}^{\circ}$ oscillometric readings, is evidence of an effective collateral system. When, on the other hand, there is a high resistance in a poorly developed collateral ${ }_{-}^{\circ}$ system, there will be a low blood pressure in the $\vec{\omega}$ arteries beyond the block; this may be demon-o strable by elevating the limb, when the digits willo blanch if the pressure is insufficient to raise the 3 . blood against gravity.

Finally, the disposition and size of collateral arteries can be displayed by arteriography. ON

\section{Conclusion}

Enough has been said to indicate features of collateral development which have clinical im- portance. Evolution of collateral systems is $a_{\mathbb{D}}$ inevitable sequel of arterial block if blood con- $-\frac{\mathbb{D}}{\mathbb{D}}$ tinues to flow; the physical changes brought 3 about by the block accelerate the blood flow through the collateral pathways, which stimula scauses these pathways to enlarge and becores transformed into collateral arteries. To assist this. $\omega$ process, measures to increase the speed of blootd flow, to remove vaso-constrictor tone and to prevent spread of thrombosis are likely to be helpful.

At the time of an acute block the stimulus to $\frac{2}{2}$ collateral enlargement is necessarily great and, $\stackrel{\varrho}{\overrightarrow{7}}$ provided the flow is adequate for the survival of $\frac{0}{3}$ resting tissues and further thrombosis does not interfere, rapid improvement follows. As the collaterals accommodate themselves to the low flows prevailing in resting tissues the stimulus 3 . wanes but is augmented again if the blood flow is increased by muscular activity, when collateral 3 . development may be expected to proceed to a new level. Regimes intended to maintain high blood flows in the limb may procure further improve-o ment; these may be based on warmth, both local? for its vaso-dilator effect and general to inhibito thermo-regulatory vaso-motor tone, on exercise regularly and frequently repeated, together witho sufficient rest and sleep and perhaps vaso-dilatori drugs. In certain patients further development ofN collateral systems, sufficient to overcome marginaf $f_{0}^{\omega}$ deficits in the circulation, may be achieved by suitable sympathectomy and confer great? symptomatic benefit.

Bibliography on page 454 
and its onset is in adolescence or early adult life, often associated with a history of similar attacks in the siblings. A reactive hyperaemia test should always be performed. The hand is immersed to the wrist in water at $40^{\circ} \mathrm{C}$. and vascular occlusion maintained for five minutes by a pneumatic cuff at a suprasystolic pressure. At the end of this time the hand is removed from the water, dried and the fingers massaged white. On release of the cuff the pink flush should spread evenly to the tips of the digits within three to four seconds. When digital artery thromboses or narrowing has occurred or should the digital arteries fail to relax, then a delay or assymmetry of return of the flush will be evident, implying that the case is not one of the idiopathic group. An E.S.R. and blood count are routinely performed together with a radiological examination of the wrists and hands for joint change and of the shoulder girdle for the presence of a cervical rib element.

\section{Treatment}

To cure or alleviate R.P. it would be necessary either to change the response of the digital arteries to cold (the local fault) or, in cases with occlusive vascular disease, to increase the resting blood flow to the digits. We know of no way of influencing the local fault although the effects of cortisone are being explored experimentally. Some increase in the resting blood flow to the fingers, and therefore rather slower cooling, can be obtained by a sympathetic denervation or by the administration of vasodilator drugs. The hand blood flow following sympathectomy is only significantly increased for a few weeks (Walker, Lynn and Barcroft, 1950) and the digital vessels quickly regain their preoperative tone and, in accordance with Cannon and Rosenbleuth's ' law of denervation,' may, in fact, become over-sensitive to circulating adrenaline and nor-adrenaline. Results of the operation in severe cases or where a marked local fault is present are therefore disappointing. It is not unusual to see a ' relapse' within a week of operation in the collagen group of cases. In patients with obliterative vascular disease the clinical response is more satisfactory and healing of the nutritional lesions often results. The type of sympathectomy preferred is the anterior (Telford) approach $\frac{\varrho}{z}$ through which the second and third thoracic $\underset{\mathbb{Q}}{\stackrel{2}{2}}$ ganglia are removed on both sides at the same $\frac{\varrho}{c}$ operative session. The removal of the stellate ganglion does not improve the operative results and $\stackrel{\vec{F}}{\rightarrow}$ causes considerable distress from the resultant $\bar{C}$ ptosis and nasal congestion. Removal of a cervical $\frac{\bar{\sigma}}{\bar{*}}$ rib where this is the causative agent will relieve the $\frac{\bar{\omega}}{\overrightarrow{0}}$ R.P. Workers who develop vibration R.P. should $\stackrel{\Phi}{\Phi}$ abandon the use of the vibration tools before the attacks become severe.

The vasodilator drugs, many of which are of $\vec{\circ}$ great experimental and pharmacological interest, $\overrightarrow{\vec{\omega}}$ have proved very disappointing in clinical practice. None of them act selectively on the digits or: limbs and their ganglionic or adrenergic blocking 3 . properties have general effects on the cardio-i vascular system, the gastrointestinal tract, the $\omega_{\omega}$ pupils and secretory glands. In addition many of the drugs have multiple pharmacological pro- $\mathrm{i}$ perties; thus Tolazoline B.P. (Priscoline) has pilocarpine and histamine-like actions. In general, 음 although the mild cases may benefit from Tola- zoline, its administration is frequently accom- $\mathbb{D}$ panied by unpleasant side effects such as dizziness, $\frac{\mathbb{O}}{\mathbb{D}}$ nausea, flushing and palpitations.

\section{Summary}

I. The production of the colour changes curring in Raynaud Phenomenon has been cussed.

2. Clinically the patients have been grouped into (a) primary or idiopathic; (b) secondary to obliterative vascular disease; (c) secondary to the development of a ' local fault.'

3. The differentiation of the above cases has $\stackrel{2}{\Rightarrow}$ been described.

4. The limitations of sympathectomy and vaso- $-\frac{3}{2}$ dilator drugs in the treatment of Raynaud Phe-O nomenon have been discussed.

\section{BIBLIOGRAPHY}

CANNON, W. B., and ROSENBLEL'TH, A. (1949), "The Supersensivity of Denervated Structures.' New York, Macmillen.

LEWIS, T. (1932) Brit. med. F., 2, 136.

LEWIS, T., and PICKERING, G. W. (1934), Clin. Sci., 1, 327.0 WALKER, A. J., LYNN, R. B., and BARCROFT, H. (1950), St. Thom. Hosp. Rep., 6, 18.

Continued from page 458-Collateral Circulation in the Limb, by C, F. Longland:

\section{BIBLIOGRAPHY}

DE BAKEY, M., and SIMEONE, F. A. (1946), Ann. Surg., 123, 534. DORNHORST, A. C., and SHARPEY-SCHAFER, E. P. (1951), Clin. Sci., 10, $37 \mathrm{I}$.

ECKSTEIN, R. W., GREGG, D. E., and PRITCHARD, W. H. (1941), Amer. F.' Physiol., 132, 35 I.

HUGHES, A, F, W, (1935), f. Anat. Lond., 70, 76.
HUGHES, A. F. W. (1937), Ibid., 72, I.

SALMON, M. (1933), "Artères des Muscles des Membres et dư⿱ Tronc,' Masson et Cie., Paris.

SALMON, M. (1936), 'Artères de la Peau,' Masson et Cie, Paris $\frac{\mathrm{C}}{\mathbb{D}}$ SALMON, M. (1938), Marseille Méd., 76 /I/, 433.

THEIS, F. V. (1933), Surg. Gynec. Obstet., 57, 737.

WOOLLARD, H. H., and WEDDELL, G. (1934), F. Anat. Lond. O 69, 25. 